\title{
Paper ID: 8075 \\ Fabrication and Packaging Technologies of Love-wave-based Microbalance for Fluid Analysis
}

\author{
L. EL Fissi ${ }^{*, a}$, J-M. Friedt ${ }^{\mathrm{a}}$, F. Chérioux ${ }^{\mathrm{b}}$, V. Luzet ${ }^{\mathrm{b}}$, S. Ballandras ${ }^{\mathrm{a}} \mathrm{b}$ \\ ${ }^{a}$ SENSeOR, Parc de Haute Technologie Lot $n^{\circ} 3$ 694, Avenue du Docteur Maurice Donat 06250 MOUGINS \\ France \\ ${ }^{b}$ Institut FEMTO-ST, UMR 6174 CNRS-UFC-ENSMM-UTBM, Besançon, France
}

\begin{abstract}
Sensors able to operate with liquid phases are the key-point for the development of bio-chemical lab-on-chip devices. In this work, we present the design and manufacture of Love wave sensors equipped with a liquid cell, at the wafer scale level, to allow operation with liquid media. Love wave sensors are fabricated on AT-cut quartz plates (IEEE Std-176 notation (YXIt)/36/90) and passivated by a $2.5 \mu \mathrm{m}$ thick silica overlay. The fabrication process using the celebrated SU-8 epoxy-based photoresist combined with silicon or quartz machined covers is presented, revealing robust enough for ensuring a reproducible sensor fabrication and packaging. Results are reported emphasizing the efficiency of the proposed approach.
\end{abstract}

Key words: Love waves sensors, liquid cell, AT-cut quartz, SU-8 epoxy, silicon, quartz.

\section{Introduction}

Quartz crystal microbalance (QCM) based techniques have been developed for years to address various kinds of biochemical analysis in liquid media. An alternative to this approach, based on guided acoustic shear waves, the so-called Love wave devices, has been demonstrated to exhibit larger gravimetric sensitivities. However, this approach is more complicated to implement as the surface on which reactions are achieved is the same as the one used for electrical connection. As a consequence, a microfluidic set-up must be implemented to prevent unwanted interactions between the corresponding areas (InterDigitated Transducers - IDTs - and propagation path). The main issue when using Surface Acoustic Wave (SAW) Sensors for biochemical analysis in liquid media [1]-[4], especially in a commercial objective, is the development of a reliable and reproducible fluidic system [5] meeting the main following requirements:

${ }^{*}$ Corresponding author Tel:+33 381402459 Fax:+33 381885714

Email address: lamia.elfissi@femto-st.fr (L. EL Fissi ) 
i) low acoustic leakage. ii) chemically inert to biological samples. iii) reproducible fabrication at the wafer scale level.

In the present work, we develop two packaging approaches for SAW sensors using the SU-8 epoxy-based photoresist. In the first one, the SU-8 is patterned directly on the silica guiding layer, preventing liquids from covering the IDTs while defining the chemical reaction area in the region between the IDTs. In the second approach, we explore the use of the SU-8 epoxy-based photoresist combined with silicon or quartz machined covers for the fabrication of this fluidic circuit. The structure is fabricated using Deep RIE chemical etching, the cover is then glued using a thin glue layer. In this case, the packaging system prevents covering the IDTs with liquids and defines the sensing area in the region in-between the IDTs. Following a detailed description of the fabrication process, we analyze the influence of the proposed packaging on the device operation by recording the evolution of the magnitude and the phase of its transfer function for each configuration considered in order to define an optimal packaging architecture.

\section{Structure description of the Love-wave-based microbalance}

Love-wave devices consist in delay lines built on 4-inch diameter, $380 \mu \mathrm{m}$ thick (AT) cut quartz wafers. The wave guidance is achieved by depositing a $2.5 \mu \mathrm{m}$ thick silica layer atop an AT-cut plate of quartz. The Love wave is excited and detected using IDTs composed of 50 pairs of 4-finger-per-wavelength electrodes made of $200 \mathrm{~nm}$ thick aluminum. The grating period is $10 \mu \mathrm{m}$, i.e. a wavelength of $40 \mu \mathrm{m}$, yielding a frequency operation in the vicinity of $125 \mathrm{MHz}$. A $3.2 \mathrm{~mm}$ long cavity is defined between the two IDTs, corresponding to the location where biochemical reactions are assumed to take place (the so-called sensing area) and the acoustic aperture is $3.5 \mathrm{~mm}$. Figure 1 displays a schematic of the delay line configuration and figure 2 shows a picture of the dual (differential) delay line once packaged on an FR4 printed circuit board.

Figure 3 shows the typical response of a silica based Love-wave delay line before packaging: insertion losses are nearly $23 \mathrm{~dB}$, with a baseline lower than $50 \mathrm{~dB}$. The ripples near the band pass center are due to parasitic reflections on the device edges.

\section{Microfluidic Packaging}

One important challenge for the development of biosensor consists in their packaging. Liquids used for bio-sensing experiments dramatically affect the transducing efficiency while in contact with the IDTs.

In order to solve this problem, we propose the fabrication of a liquid cell isolating the sensing area and the IDTs along two technological approaches. The first solution consists in patterning a SU-8 based liquid cell atop the $\mathrm{SiO}_{2}$ guiding layer. For the second solution, we propose a sensor structure for which a cover is machined and bonded to the quartz sensor. Both are manufactured separately, and then glued together atop the SAW devices using a thin SU-8 film deposited on the cover plate.

For both solutions, two principal aspects have been considered for the design of the liquid cell. First, we have selected an open well configuration for an easy access to the 
sensing area. Second, the packaging must cover the smallest length on the acoustic path to minimize acoustic perturbations while still ensuring the best liquid tightness confinement. In order to evaluate the actual impact of the structure on the wave propagation, five wall widths $(80,100,120,140$ and $200 \mu \mathrm{m})$ have been tested. We particularly intend to evaluate the influence of this parameter on the attenuation of the acoustic wave.

\subsection{SU-8 liquid cell fabrication}

The negative epoxy-based photoresist SU-8 has been used extensively as a resin to make high aspect ratio Micro-Electro-Mechanical Systems (MEMS) device structures and packaging parts, by virtue of its good mechanical properties [7], water impermeability and dielectric nature once polymerized. A main application of SU-8 is making electroplating molds [8]-[9], injection molding masters and embossing masters [10][11] for structures with high aspect ratio. Owing to its bio-compatibility and chemical resistance, SU8 is also ideally suited for the fabrication of micro-channels for microfluidic and bioMEMS devices [12]-[13].

We present now the basic steps of the SU-8 technology developed here for the fabrication of this liquid cell. Figure 4 summarizes this fabrication process. The overall thickness of the structure is about $120 \mu \mathrm{m}$.

The adhesion of a thick SU-8 layer onto silica overlays is known to be poor if cleaning and drying procedures are not carefully respected. The SU-8 2075 resist from Microchem Corp. (MA, USA) has been used here. Prior to photoresist coating, the quartz wafer with SAW transducers must be dehydrated at $200{ }^{\circ} \mathrm{C}$ for 30 minutes (fig.4-a) in order to improve the adhesion of the SU-8 [6]. The SU-8 2075 is spread at $300 \mathrm{rpm}$ (acceleration fixed to $100 \mathrm{rpm} \mathrm{s}^{-1}$ ) during $60 \mathrm{~s}$, followed by a spin-coating at $1600 \mathrm{rpm}$ (acceleration $300 \mathrm{rpm} \mathrm{s}^{-1}$ ) during another $60 \mathrm{~s}$ in order to achieve a layer thickness of $120 \mu \mathrm{m}$ (fig.4-b). After spin coating operations, an initial soft-bake is carried out on a hotplate at $95{ }^{\circ} \mathrm{C}$ for 35 minutes to remove the photoresist solvents (fig.4-b). This layer is patterned by standard photolithographic techniques to form the walls (fig.4-c). A second bake is then performed at $95{ }^{\circ} \mathrm{C}$ for 15 minutes to finish the cross-linking of the SU-8 (fig.4-d). The unexposed epoxy is then dissolved in propylene glycol monomethyl ether acetate (PGMEA) (fig.4-e). Once these operations achieved, the wafer must be diced to release each sensor with its patterned SU-8 fluidic structure. Figure 5 presents a scanning electron microscope (SEM) image of the final SAW sensor equipped with its SU-8 based liquid cell.

The resulting sensor is glued on a FR4 epoxy printed circuit, the electrical connections being performed by a conducting silver loaded epoxy between the bonding pads and the copper tracks on the circuit.

Figures 7 and 8 show the effect of the presence of thick SU-8 photoresist walls on the acoustic path. According to these curves, we find out that the acoustic wave is significantly affected by the presence of the SU- 8 walls and the insertion loss increases when increasing their width. We also note that reflections of the acoustic waves on the walls are more pronounced as the width of the walls increases. These reflections generate ripples in the band and their spectral separation corresponds to the wave velocity divided by twice the propagation length in the sensing area between the edge of the two side walls (i.e. $5000 \mathrm{~m} \cdot \mathrm{s}^{-1} / 6.4 \mathrm{~mm} 800 \mathrm{kHz}$ ). We also observe the vanishing 
of parasitic reflections on the edges of device observed on the transfer function of the device without liquid cell.

\subsection{Silicon and quartz liquid cell fabrication}

We have used a two-step method to fabricate silicon and quartz liquid cells. Both of them are achieved at the 4-inch wafer level. The first step consists in the fabrication of SAW sensors and silicon or quartz covers separately. We then glue the cover plate on the SAW devices using a thin SU-8 film deposited on the cover plate.

\subsubsection{Silicon covers}

In order to manufacture silicon covers, we use a Deep Reactive-Ion Etching (DRIE). The process flow-chart developed here is shown in figure 6 .

The cover is etched in a double side polished silicon wafer. A $2 \mu \mathrm{m}$ thick thermal oxide is first grown on the wafer. The sensing area is then etched on the whole wafer thickness $(500 \pm 25 \mu \mathrm{m})$. The wafer is cleaned in an acid solution 15:1(v/v) $96 \mathrm{wt} \%$ $\mathrm{H}_{2} \mathrm{O}_{2}$ (so-called Piranha) for 20 minutes. After the cleaning process, the wafer is rinsed in de-ionized water and dehydrated at $200{ }^{\circ} \mathrm{C}$ for $30 \mathrm{~min}$ (fig.6-1). The $2 \mu \mathrm{m}$ thick termal oxide is used as a mask to define the IDTs cavities.

The fabrication process consists of two steps: first, a double side photolitography is carried out using the S1813 photoresist. With this resist, we define on the first side the two IDT cavities and on the second side the alignment pattern (fig.6-2). In that purpose, a S1813 photoresist film is spin coated onto the wafer at $4000 \mathrm{rpm}$ for $30 \mathrm{sec}$ and softbaked, followed by a soft contact lithography for patterning. Finally, the IDT cavities and the alignment cross are obtained by dissolving the exposed epoxy in AZ726 developer. Following the development, the wafer is etched in a Buffered Hydrofloric Acid (BHF) solution in order to remove the thermal oxide (fig.6-3). After etching the wafer, the structures on the wafer are examined using an optical microscope. Subsequently, the photoresist on the silicon wafer is stripped by immersing the wafer respectively in acetone, isopropanol and de-ionized water for 5 minutes each (fig.6-4).

For the second phase, the positive photoresist and the developer used are SPR 7.0 and AZ726 from Micochem. This photoresist is used as a second mask to define the sensing area cavity. The SPR 7.0 photoresist is spin coated onto the wafer at $2000 \mathrm{rpm}$ for $30 \mathrm{sec}$ (fig.6-5), followed by an exposition step (600 mJ). Following the development, the patterned wafer is rinsed in de-ionized water (fig.6-6). Last, the wafer is etched using DRIE. The DRIE has an approximate etch rate of $3.5 \mu \mathrm{m} / \mathrm{min}$ and the required etching delay is 70 minutes to achieve a $250 \mu \mathrm{m}$ trench depth (fig.6-7). The photoresist is stripped by immersing the wafer in acetone, isopropanol and de-ionized water in order to start the second etching step of the IDT cavities and to finish the etching of the sensing area cavity (fig.6-8). Figure 9 presents a SEM photo of the silicon cover.

\subsubsection{Quartz covers}

A wet etch process is used to manufacture the quartz cover: the corresponding process flow-chart is shown in figure 10.

A double-side polished quartz ((ZXl) $1,5^{\circ}$ ) wafer (4-inch diameter, $500 \pm 25 \mu \mathrm{m}$ thick) from Roditi International Corporation Ltd, UK is used. After cleaning the wafer 
in a Piranha solution, $10 \mathrm{~nm}$ thick chromium and $120 \mathrm{~nm}$ gold layers are deposited by electron beam evaporation onto the double side wafer (metal protection layer) (fig.10$1)$.

The S1813 photoresist is spin coated and patterned to define the IDT and sensing area cavities. A Cr/Au mask is then patterned for quartz machining (fig. 10-3). Subsequently, the wafer is etched using BHF solution with an approximate etch rate of $1.5 \mu \mathrm{m} \cdot \mathrm{min}^{-1}$. After etching the wafers, the photoresist on the quartz wafer is stripped by immersing the wafer in acetone, isopropanol and de-ionized water, following by etching the $\mathrm{Cr} / \mathrm{Au}$ protection layer (fig.10-4). Figure 11 presents a SEM view of the quartz cover.

\subsubsection{Sensors and cover assembly}

Following the fabrication steps of the SAW sensors and silicon or quartz covers, we glued the cover plate on the SAW devices. For that, both wafers are cleaned using an UV-ozone cleaner for 60 minutes to avoid particle adherence to the silicon or quartz surface. A thin SU-8 (NANO ${ }^{T M}$ SU-8 2000 Microchem) film is then deposited on the cover plate. To evaporate the solvent contained in the SU-8 film, the cover coated with the SU-8 film is baked for 5 minutes at $75^{\circ} \mathrm{C}$. After that, the two wafers are aligned and placed into an EVG bonding machine. During the bonding processing, we apply a pressure of $75 \mathrm{~N}$ on both wafers while increasing the temperature from $60^{\circ} \mathrm{C}$ to $95^{\circ} \mathrm{C}$. After 4 hours of bonding, the wafer is diced to release each sensor with its cover. Figure 12 presents a SEM image of the final SAW sensor equipped with its quartz cover.

\subsection{Evolution of the insertion loss of silicon and quartz liquid cell}

Figures 13 and 14 show the evolution of the insertion loss versus the width of the resistive silicon and quartz walls deposited on the acoustic path, measured using a Rohde \& Schwarz ZVR network analyzer.

The acoustic wave propagation is significantly affected by the presence of the silicon or quartz covers: the insertion loss increases when increasing the width of the walls supporting the covers. We also observe that the quartz cover generates slightly higher insertion loss than the silicon one.

Like SU-8 walls, quartz and silicon walls also generate acoustic reflections. On the other hand the SU-8 walls generate less insertion losses than the cover combined with the SU-8 glue for the same width. One can consider that these solid covers generate more scattering, yielding more insertion loss.

We compare (Figs. 13 and 14) the evolution of the insertion losses according to various compressive forces $(75 \mathrm{~N}, 35 \mathrm{~N}, 15 \mathrm{~N})$ applied on both wafers during the bonding step: the insertion losses decrease significantly when reducing the compressive force, as observed both for the silicon and quartz covers. The insertion losses decrease is explained by the fact that the photoresist spreading over the propagation surface is proportional to the compressive force.

The efficiency of this process has been demonstrated by using such devices as biochemical sensors, as demonstrated previously [14]. During these experiments, the liquid tightness was validated during chemical reaction processes lasting overs 4 hours with acqueous solutions and overnight functionalization in organic solvents. 


\section{Conclusion}

Two technological approaches for SAW sensor packaging have been developed. Both are compatible with wafer-level batch processes, and yield additional acoustic losses as walls are directly bonded on the acoustic wave path. The SU-8 wall approach induces the lowest additional losses, the provides no closed-capping over the IDTs, making the resulting devices difficult to use. Capping with structured quartz and silicon wafer provides full sealing of the area of the IDTs, but additional losses due to the epoxy spreading on the acoustic path during the wafer bonding step.

\section{Acknowledgements}

The authors would like to thank G. Heider (SENSeOR) for his help in promoting this work.

\section{References}

[1] E. Gizeli, in Biomolecular Sensors, Chapter on "Acoustic immunosensors", Eds E.Gizeli \& C.R. Lowe, 2002, Taylor \& Francis, UK.

[2] T.M.A. Gronewold, "Surface acoustic wave sensors in the bioanalytical field: Recent trends and challenges", Anal. Chim. Acta, vol. 603, pp. 119-128, 2007.

[3] B. Jakoby and M. Vellekoop, "Viscosity sensing using a love-wave device", Sensors and Actuators A, vol. 68, pp. 275-281, 1998.

[4] K.Länge, G. Blaess, A. Voigt, R. Götzen, M. Rapp, "Integration of a surface acoustic wave biosensor in a microfluidic polymer chip", Biosensors and bioelectronics, vol. 22, pp. 227-232, 2006

[5] V. Raimbault, D. Rebière, C. Dejous, M. Guirardel, "Acoustic love wave platform with PDMS microfluidic CHIP", Sensors and Actuators : A Physical, vol. 142, pp. 160-165, 2007.

[6] H. Lorenz, M.Despont, N. Fahrni, N. Lablance, P. Renaud, P. Vettiger, "SU-8: A low cost negative resist for MEMS", J. Micromech. Microeng, vol. 7, pp. 121124, 1997

[7] H. Lorenz, M. Laudon, P. Renaud, "Mechanical Characterization of a new ighaspect-ratio near UV-photoresist", Microelectronic Engineering, vol. 41-42, pp. 371-374, 1998.

[8] L.J. Guerin, A. Torosdagi, P. Eichenberger, P. Renaud, N. M. White, "High aspect ratio planar coils embedded in SU8 photoepoxy for MEMS applications", Eurosensors XII, vol. 1, pp. 11-14, 1998.

[9] V. Seidemann, S. Bttgenbach, "A novel fabrication process for 3D meander shaped micro coils in SU8 dielectric and their application to linear micro motors", SPIE proceedings series, vol. 4407, pp. 304-309, 2001. 
[10] H. Lorenz, M.Despont, P. Vettiger, P. Renaud, "Fabrication of photoplastic high aspect ratio microparts and micromolds using SU8 UV reist", Microsystem Technologies, vol. 4, pp. 143-146, 1998.

[11] J. Zhang, K. L. Tan, G. D. Hong, L. J. Yang, H. Q. Gong, "Polymerization optimization of SU-8 photoresist and its applications in microfluidic systems and MEMS”, Micromechanics and Microengineering, vol. 11, pp. 20-26, 2001.

[12] L. Che-Hsin, L. Gwo-Bin, C. Bao-Wen, C. Guan-Lian, "A new fabrication process for ultra-thick microfluidic microstructures utilizing SU-8 photoresist", Micromechanics and Microengineering, vol. 12, pp. 590-597, 2002.

[13] L. A. Francis, J.-M.Friedt, C. Batric, A. Campitelli, "A SU-8 cell for surface acoustic wave biosensors", SPIE proceedings series, vol. 5455, p. 353-363, 2004.

[14] L. EL Fissi, J.-M. Friedt, V. Luzet, F. Cherioux, G. Martin, S.Ballandras, “A lovewave sensor for direct detection of biofunctionalized nanoparticles", Frequency Control Symposium, 2009 Joint with the 22nd European Frequency and Time forum. IEEE International, vol. 20, pp:861-865, 2009.

\section{biographies}

Lamia El-Fissi was born in Casablanca (Marocco) in 1980. She received her PhD from University of Franche-Comte in Besancon (France) in 2009. Her current research interests are design, fabrication and development of SAW sensors and micro-fluidic devices for applications of lab-on chip devoted to biology.

Dr. J.-M Friedt obtained his PhD from University of Franche-Comte in Besancon (France) in 2000. Following a 3 years position as a postdoctoral fellow with the Biosensors group in IMEC (Leuven, Belgium), he joined the team of S. Ballandras at the FEMTO-ST institute in Besancon (France) and is currently employed by the company SENSeOR. His interests include direct detection biosensors using optical and acoustic methods, scanning probe microscopy, and especially the combination of these techniques for multiparametric data analysis.

Dr. Frederic Chérioux obtained a PhD in Physical Chemistry from the University of Franche-Comté in 1999 and an habilitation degree from the same University in 2005. He was a researcher at the Institute of Chemistry in Neuchatel (Switzerland) from 2000 to 2003 in the Suss-Fink's Group. He is CNRS researcher at the FEMTO-ST institute in Besanon since 2003. His research is focused on the physics and chemistry of isolated molecules and supramolecular self-assembly on semiconductor.

Vincent Luzet is specialized in organic synthesis. He had been working as an intern for three months at the University of Montreal (Canada) in 2006 and 8 months at the University of Turku (Finland) in 2007. He is currently, and since 2008, a CNRS engineer assistant at the FEMTO-ST Institute (France).

Dr. Sylvain Ballandras joined the CNRS in 1991, after his PhD in Engineering Sciences from the Universit de Franche-Comt. Until 1995, he has been working on SAW devices, but he was also involved in microtechnologies. He has initiated the development of a finite element analysis package devoted to acoustic transducers and also new researches on miniaturizedtransducers. During 1997, he achieved a one year industrial training project in SAW industry. In October 2003, he was promoted Research Director at the CNRS. In 2008 , his group joined the Time-Frequency Department and moved to the national engineering school ENSMM. Its present scientific developments concern fundamentals in acoustics and guided propagation as well as technologies dedicated to acousto-electronic devices and systems, focused on sources, filters and sensors. Sylvain Ballandras also benefits from the 25.2 agreement of the French research rules to join the head staff of SENSeOR since the end of 2008. 


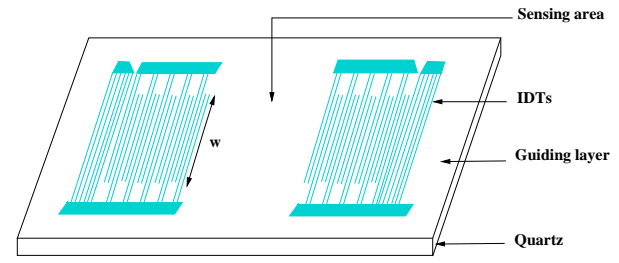

Figure 1: Scheme of a Love-wave based delay line.

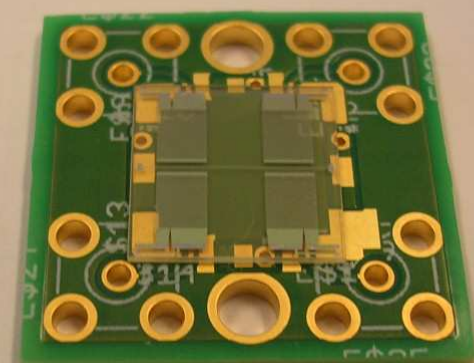

Figure 2: Picture of the dual delay lines device glued on a FR4 epoxy printed circuit (26*26 mm).
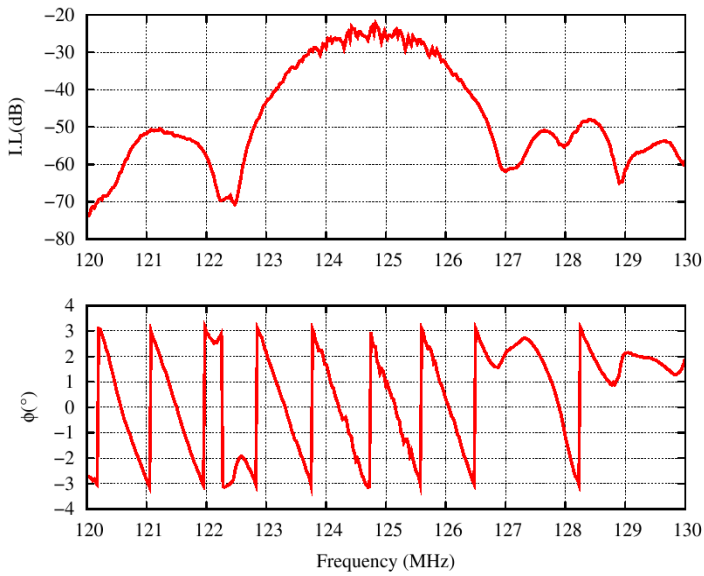

Figure 3: Typical transfer function of delay line exploiting silica guiding overlays. 
(a)

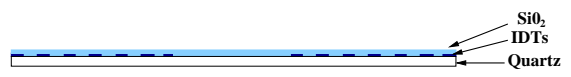

(b)

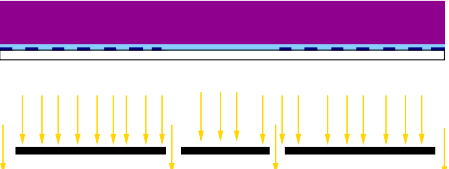

(c)
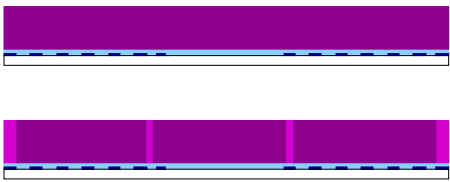

(e)

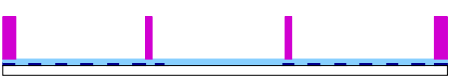

Figure 4: SU-8 walls fabrication steps. (a) Love wave delay line with $\mathrm{SiO}_{2}$ guiding layer ; (b) spin coating of SU8 2075 and soft-bake ; (c) soft contact lithography ;(d) post-bake ; (e) development of SU-8.

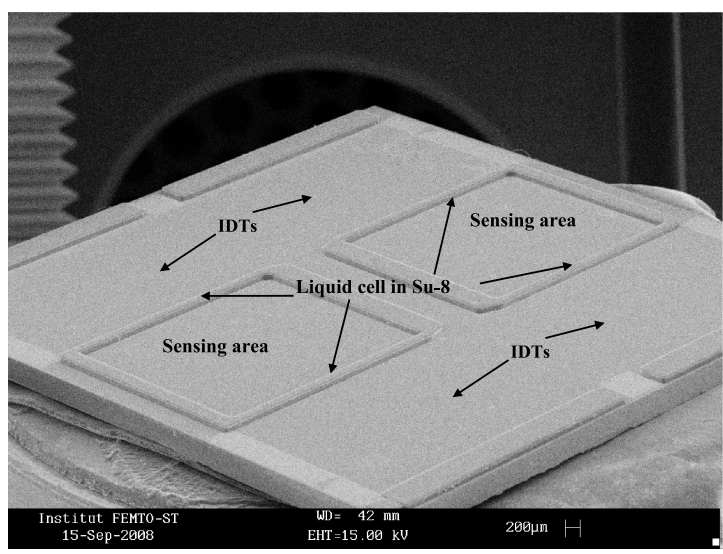

Figure 5: SEM image of the SAW device coated with a patterned thick SU-8.

2)

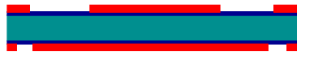

3)

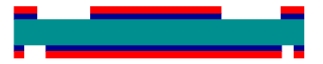

4)

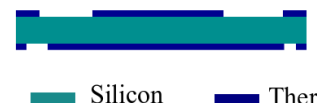

5)

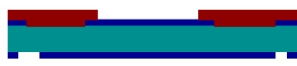

6)
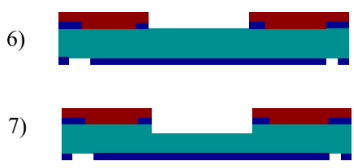

8)

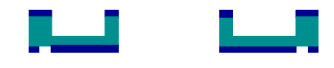

Figure 6: Fabrication process of the silicon cover. 


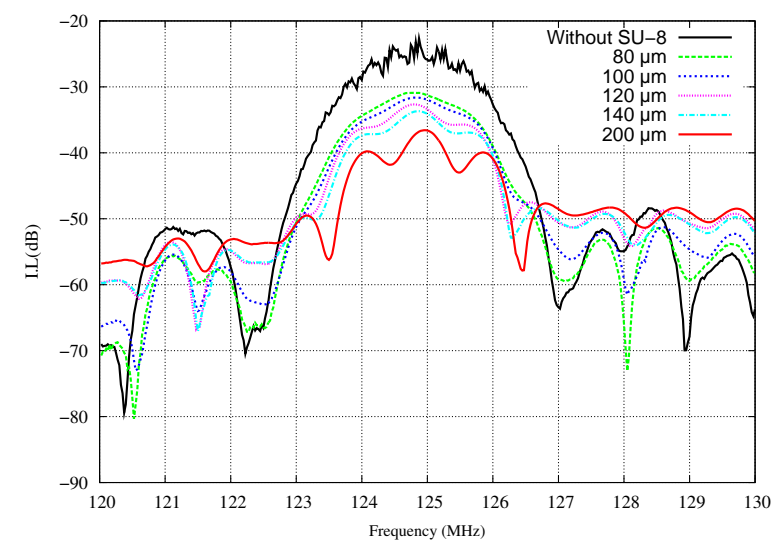

Figure 7: Evolution of the insertion loss versus the width of the walls deposited on the acoustic path.

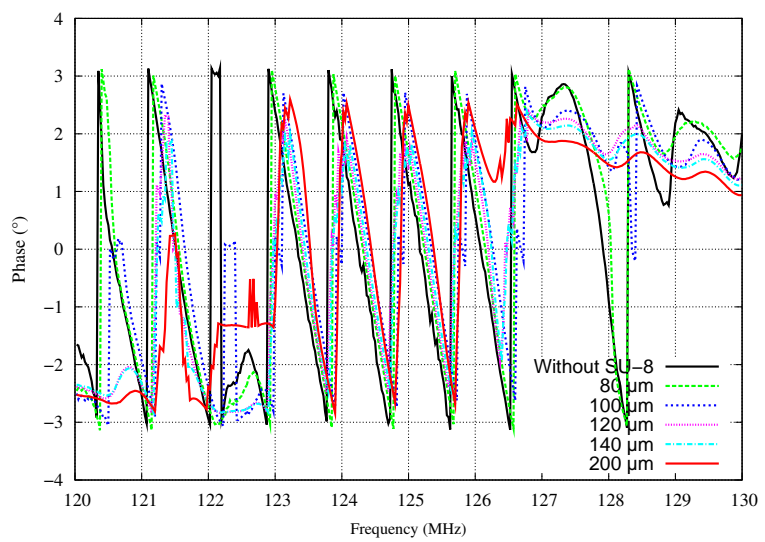

Figure 8: Evolution of the phase versus the width of the walls deposited on the acoustic path.

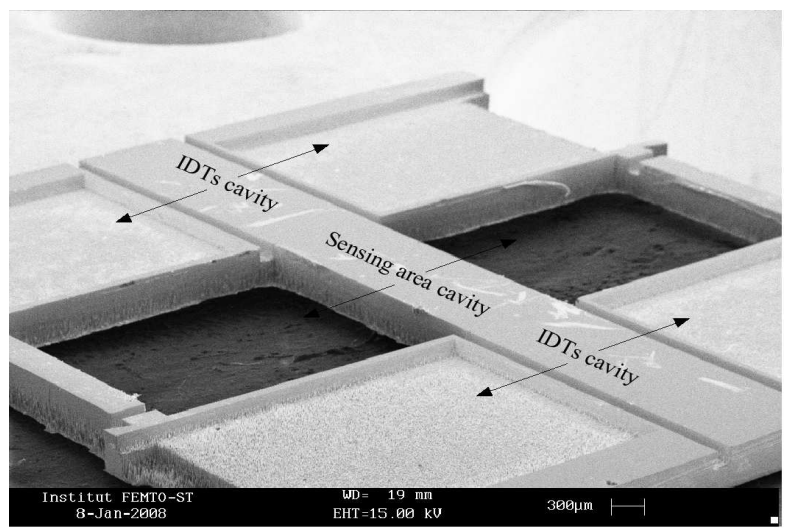

Figure 9: SEM image of silicon cover. 
1)

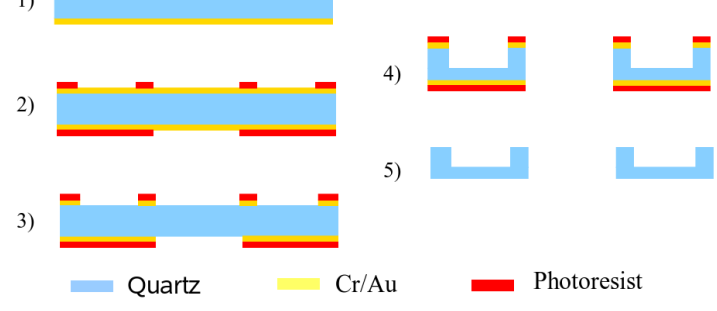

Figure 10: Quartz cover fabrication process flowchart.

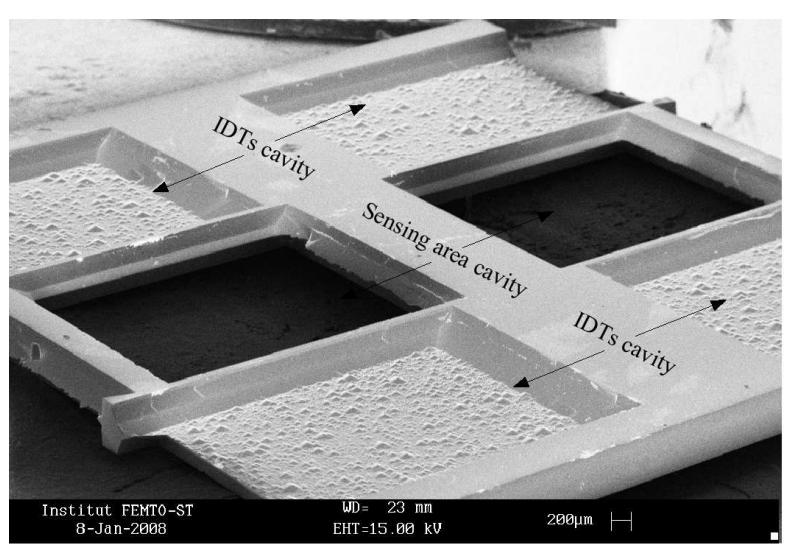

Figure 11: SEM image of quartz cover.

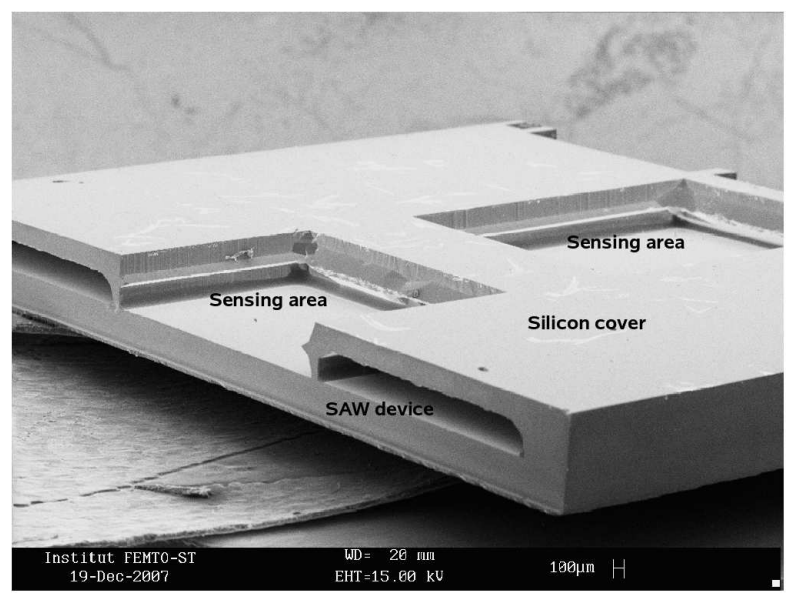

Figure 12: SEM image of the SAW sensor with quartz cover. 


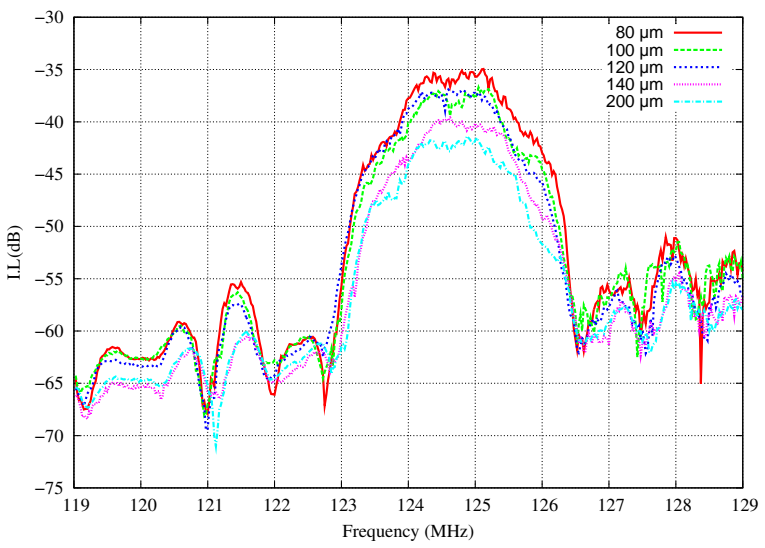

Figure 13: Evolution of the insertion loss versus the width of the silicon walls supporting the covers with a $75 \mathrm{~N}$ compressive force.

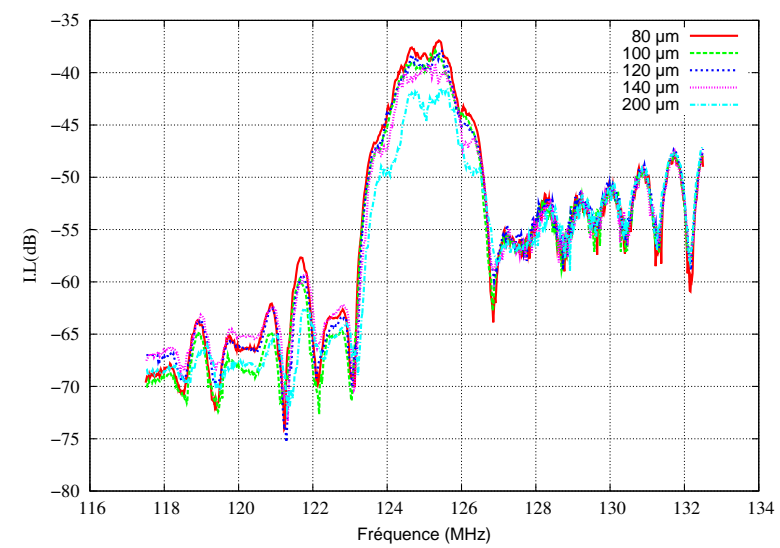

Figure 14: Evolution of the insertion loss versus the width of the quartz covers with a $75 \mathrm{~N}$ compressive force. 


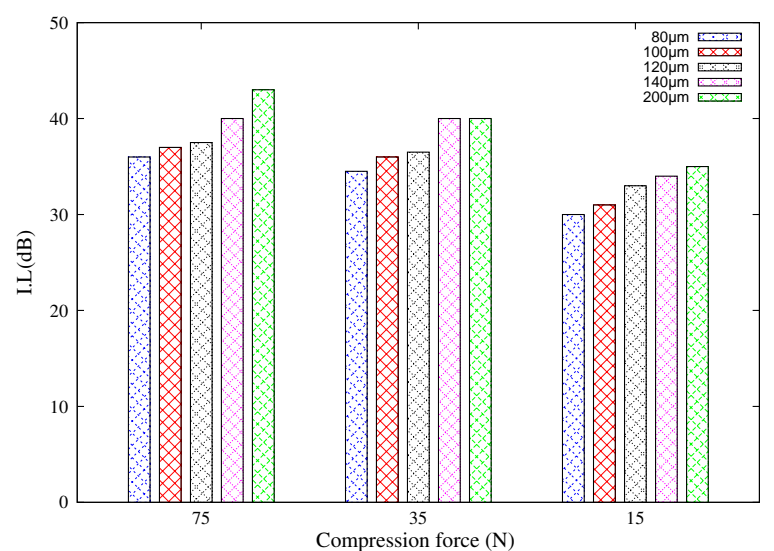

Figure 15: Histogram of the insertion losses according to various compressive forces applied to bond the silicon covers.

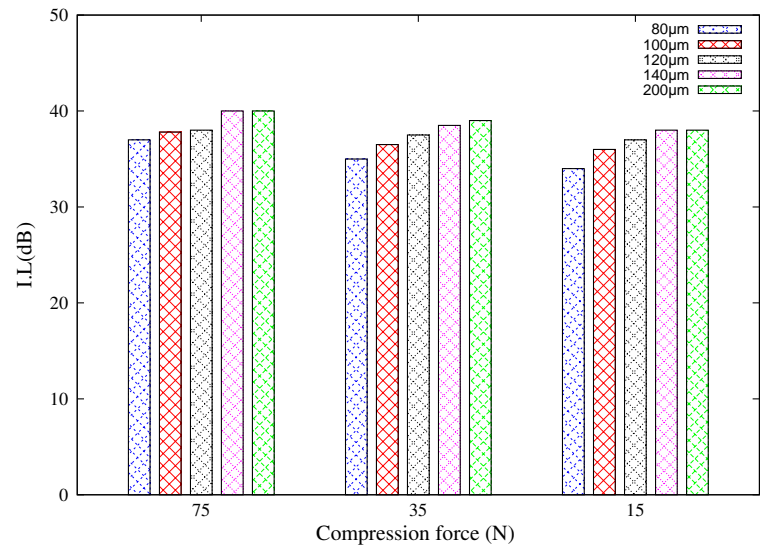

Figure 16: Histogram of the insertion losses according to various compressive forces applied to bond the quartz covers. 